\title{
Acute postoperative pain after arthroscopic rotator cuff surgery: A review of methods of pain assessment
}

\author{
Jacob Korsbæk Rasmussen ${ }^{1}$, Lone Nikolajsen ${ }^{2}$, and Karen Toftdahl Bjørnholdt ${ }^{3, *}$ \\ 1 Aarhus University, Vennelyst Boulevard 4, Aarhus, Denmark \\ 2 Department of Anesthesiology and Intensive Care, Aarhus University Hospital, Research, C319, Palle Juul Jensens \\ Boulevard 99, Aarhus, Denmark \\ ${ }^{3}$ Department of Orthopedic Surgery, Horsens Regional Hospital, Sundvej 30, Horsens, Denmark
}

Received 22 April 2018, Accepted 10 August 2018, Published online 22 November 2018

\begin{abstract}
Introduction: Pain can be severe during the first days after arthroscopic surgery, and acute pain is an important outcome in clinical trials of surgical technique or anaesthetic strategy. A standardized, validated method of assessing acute postoperative pain would improve the quality of clinical studies, and facilitate systematic reviews and meta-analyses. A step on the way towards this standard is to investigate the methods most commonly used in recent literature.

Methods: PubMed and CINAHL databases were searched, including studies of arthroscopic rotator cuff surgery with a primary pain-related outcome during the first postoperative week, published in English from 2012 to 2017.

Results: A total of 47 studies were included, all measuring pain intensity using a pain rating scale. Most frequently used was the visual analogue scale using the anchors "no pain" and "worst pain imaginable," with recordings at $1,2,4,6,8,12$, and 24 hours postoperatively. A total of 34 studies recorded analgesic consumption, usually as average cumulated consumption in $\mathrm{mg}$. Time to first analgesic request or first pain were recorded in 11 studies, and 4 different starting points were used.

Discussion: This review describes the currently most common methods of assessing acute postoperative pain in clinical trials of arthroscopic shoulder surgery involving rotator cuff repair, and the large variety of methods applied. Based on this study and international guidelines, several recommendations on how to measure and report postoperative pain outcomes in future trials are proposed.
\end{abstract}

Key words: Rotator cuff surgery, Postoperative pain, Pain intensity, Pain rating scale, Analgesic consumption.

\section{Introduction}

Pain assessment is fundamental in orthopedic surgery to evaluate disease severity as well as postoperative improvement. When evaluating new surgical interventions and analgesic methods, clinical trials often use acute postoperative pain as an outcome. Recent quality improvement guidelines of acute postoperative pain from the American Pain Society [1], a review [2] and a study protocol [3] of acute postoperative pain after shoulder surgery have defined outcomes to be included in postoperative pain trials, such as "pain intensity" $[4,5]$. However, recommendations for outcome measurements and recordings are lacking [6]. For example, pain intensity

\footnotetext{
*Corresponding author: karenbjo@rm.dk
}

may be reported through various pain rating scales. As a result, trials have assessed postoperative pain in a wide variety of ways $[2,7,8]$.

A standardized, validated method of assessing acute postoperative pain would improve the quality of clinical studies and facilitate future systematic reviews and metaanalyses. Thus, the aim of this study was to investigate the methods used in recent literature, focusing on ratings of pain intensity, time points and number of measurements, analgesic consumption, and time to first analgesic request or first pain.

\section{Materials and methods \\ Data sources and searches}

The databases PubMed and CINAHL were used to identify studies on shoulder surgery and postoperative pain. Subject-specific terms relating to any type of shoulder 
surgery were identified in the MeSH tree, PubMed, and in Headings, CINAHL. Any subject-specific terms unavailable in the indexed headings were searched as free text in title and abstract, such as subacromial decompression. All relevant MeSH terms/headings not exclusive to surgery or pathology of the shoulder, such as arthroscopy or bursitis, were combined with a shoulder-related MeSH term/ heading. For MeSH terms introduced in 2012 or later, the previous MeSH term indexing was added to the search. To include articles with incomplete indexing processes in PubMed, a free text search in title and abstract was conducted for the last 2 years, using the subject-specific terms.

\section{Selection of included studies}

The identified articles from the systematic literature search were imported to Covidence, a web-based systematic review software (Veritas Health Innovation, Melbourne, Australia) [9]. Two reviewers, JKR and KTB, assessed the articles individually, and consensus was sought through discussion in case of disagreement. The inclusion criteria were: shoulder surgery, report of any kind of pain assessment within the first postoperative week, and published in English from 30 June 2012 to 30 June 2017. As this search provided more relevant studies for inclusion than first anticipated, this review focused on arthroscopic shoulder surgery including rotator cuff surgery, and pain as the primary outcome. If no primary outcome was specified, the first outcome mentioned in the study's material and methods section was considered primary. Finally, the bibliographies of the included studies and related review articles were manually reviewed for other relevant studies, which were then included if they met the inclusion criteria.

\section{Data collection}

From the included studies, the following data was recorded: first author, year of publication, study design, surgical procedure, intervention, total number of patients, primary outcomes, and pain-related outcomes. Details regarding the methods of pain assessment were recorded as follows: data collection by staff (telephone or personal interviews) or by patients themselves (pain diaries or questionnaires), pain scale used, phrasing of pain anchors, measurement at rest or during activity, measurement of present/average/worst pain, time-points of measurements on postoperative day (POD) 1 and number of measurements daily until POD7, analgesic consumption, time to first analgesic request or first pain.

\section{Results}

A total of 711 non-duplicate articles eligible for screening of title and abstract were identified, of which 153 were further assessed in full text; 47 studies met the inclusion criteria (Figure 1). The 47 included studies included randomized controlled trials (RCTs) $(n=33)$, non-randomized comparative studies $(n=6)$, quasi-RCT

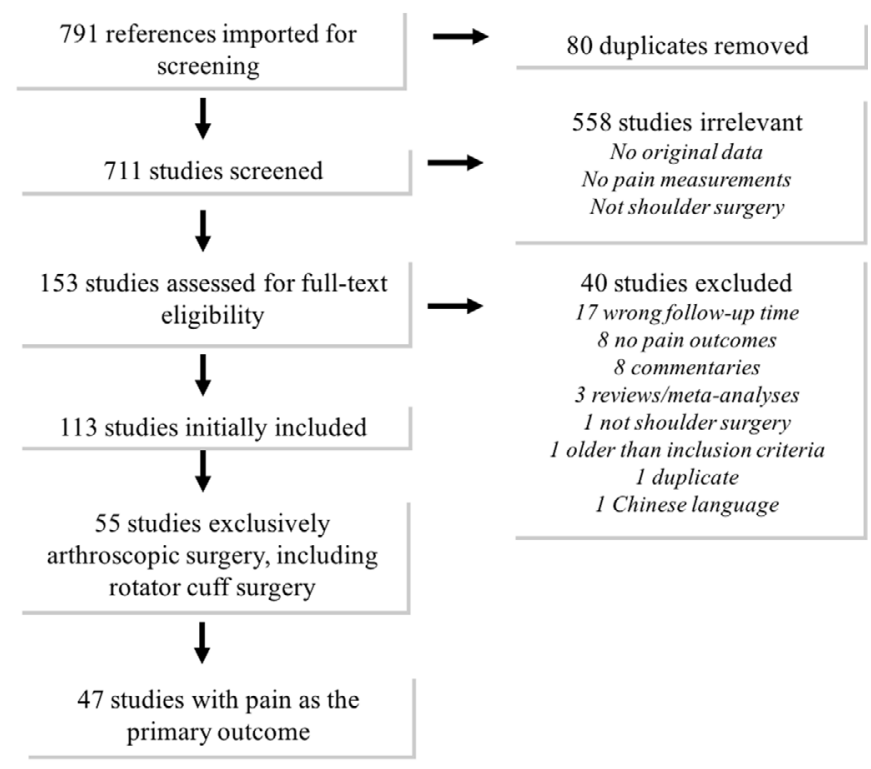

Figure 1. Flowchart.

$(n=1)$, cohort studies $(n=4)$, case series $(n=2)$, and one up-and-down dose finding study (Table 1). The study populations were patients undergoing arthroscopic rotator cuff surgery exclusively $(n=29)$ or in combination with other arthroscopic shoulder surgery $(n=18)$. The number of patients analyzed in the studies ranged from 24 to 1624 (mean 122). Interventions (Table 1) were performed by orthopedic surgeons, anesthesiologists, physiotherapists, nurses, and industry pharmacists.

\section{Primary outcome}

Pain intensity was measured using a rating scale in all 47 studies (Table 1), and was the primary outcome in 37 studies. Analgesic consumption was reported in 34 studies and was the primary outcome in 6 studies [10-15]. The time to first pain or analgesic request was reported in 11 studies and was the primary outcome in 5 studies [10,16-19] (one of which also had analgesic consumption as a primary outcome [10]). In 8 studies, a rescue analgesic was given based on pain scale ratings [10-12,15,17,20-22].

\section{Methods for data collection}

Staff-administered pain questionnaires were applied in 27 out of 47 included studies [11-14,16-21,23-39]. Nine studies $[10,18,39-45]$ used a patient-administered pain questionnaire, and 12 studies [15,46-56] did not specify their method. One study used a staff-assessed method in the hospital and a self-assessed method after patient discharge [18].

\section{Ratings of pain intensity}

Of the 47 studies, 26 used the visual analog scale (VAS), 17 used the numeric rating scale (NRS), 4 used a Likert scale, and a single study used a combined VAS and Faces Pain Scale (Table 1). 
Table 1. The 47 included studies containing arthroscopic rotator cuff surgery with pain as the primary outcome.

\begin{tabular}{|c|c|c|c|c|c|}
\hline \multirow[b]{2}{*}{ Author, year } & \multirow[b]{2}{*}{ Study design } & \multirow[b]{2}{*}{ Intervention } & \multicolumn{3}{|c|}{ Pain-related outcomes } \\
\hline & & & Pain scale & $\begin{array}{l}\text { Analgesic } \\
\text { consumption }\end{array}$ & $\begin{array}{l}\text { Time to } \\
\text { first request }\end{array}$ \\
\hline Abdallah et al., 2016 & $\mathrm{RCT}$ & $\begin{array}{l}\text { ISB + dexmedetomidine adjuvant vs. } \\
\text { IV vs. none }\end{array}$ & VAS & + & + \\
\hline Ahn et al., 2016 & $\mathrm{RCT}$ & Pregabalin vs. placebo before surgery & NRS & + & - \\
\hline Aksu et al., 2015 & $\mathrm{RCT}$ & $\begin{array}{l}\text { ISB vs. intraarticular bupivacaine vs. } \\
\text { none }\end{array}$ & VAS & + & + \\
\hline Alemanno et al., 2014 & Non-randomized & $\begin{array}{l}\text { MIB levobupivacaine vs. } \\
\text { levobupivacaine }+ \text { buprenorphine vs. } \\
\text { levobupivacaine }+ \text { tramadol }\end{array}$ & VAS & - & + \\
\hline Alemanno et al., 2016 & Non-randomized & $\begin{array}{l}\text { MIB levobupivacaine vs. } \\
\text { levobupivacaine }+ \text { thiamine }\end{array}$ & VAS & - & + \\
\hline Alfuth et al., 2016 & $\mathrm{RCT}$ & Cold compression vs. cold pack & VAS & - & - \\
\hline Basat et al., 2016 & Case-series & $\begin{array}{l}\text { Suprascapular and axillary nerve } \\
\text { block }\end{array}$ & VAS & - & - \\
\hline Cheng et al., 2016 & Cohort & $\begin{array}{l}\text { Correlation of fibromyalgia and } \\
\text { postoperative pain }\end{array}$ & $\begin{array}{l}\text { 10-point } \\
\text { scale }\end{array}$ & + & + \\
\hline Cho et al., 2015 Feb & Non-randomized & ISB vs. none & VAS/Faces* & + & - \\
\hline Cho et al., 2015 May & Non-randomized & Zolpidem vs. none & VAS & + & - \\
\hline Choi et al., 2015 & $\mathrm{RCT}$ & Stellate ganglion block vs. none & VAS & + & - \\
\hline Cuff et al., 2016 & Cohort & $\begin{array}{l}\text { Correlation of preoperative factors } \\
\text { and postoperative pain }\end{array}$ & VAS & - & - \\
\hline D'Ambrosi et al., 2016 & $\mathrm{RCT}$ & $\begin{array}{l}\text { Platelet rich plasma during surgery } \\
\text { vs. none }\end{array}$ & VAS & - & - \\
\hline Desmet et al., 2015 & $\mathrm{RCT}$ & dexamethasone IV vs. placebo & Likert & + & + \\
\hline Dhir et al., 2016 & $\mathrm{RCT}$ & $\begin{array}{l}\text { Suprascapular and axillary nerve } \\
\text { block vs. ISB }\end{array}$ & NRS & + & - \\
\hline Erden et al., 2017 & quasi-RCT & $\begin{array}{l}\text { Standard pain assessment protocol } \\
\text { vs. routine pain assessment }\end{array}$ & VAS/NRS & + & - \\
\hline Faria-Silva et al., 2016 & $\mathrm{RCT}$ & $\begin{array}{l}\text { Block with clonidine vs. block } \\
\text { without clonidine }\end{array}$ & NRS & + & - \\
\hline Han et al., 2013 & $\mathrm{RCT}$ & $\begin{array}{l}\text { Multimodal local injection vs. i.v. } \\
\text { patient-controlled analgesia }\end{array}$ & VAS & + & - \\
\hline Jo et al., 2014 & $\mathrm{RCT}$ & Multimodal local injection vs. placebo & VAS & + & - \\
\hline Khashan et al., 2016 & $\mathrm{RCT}$ & $\begin{array}{l}\text { Preincisional intraarticular morphine } \\
\text { vs. ketamine }+ \text { morphine vs. placebo }\end{array}$ & NRS & + & - \\
\hline Kim et al., 2016 & $\mathrm{RCT}$ & $\begin{array}{l}\text { ISB } 0.2 \% \text { ropivacaine vs. ISB } 0.75 \% \\
\text { ropivacaine vs. cervical epidural block }\end{array}$ & VAS & + & - \\
\hline Kraeutler et al., 2015 & $\mathrm{RCT}$ & Compressive cryotherapy vs. ice & VAS & + & - \\
\hline Lane et al., 2014 & case series & $\begin{array}{l}\text { Same day discharge after GA and } \\
\text { ISB }\end{array}$ & NRS & - & - \\
\hline Lee et al., 2015 & $\mathrm{RCT}$ & $\begin{array}{l}\text { Local anesthetic injection in the GH } \\
\text { vs. the SA vs. both }\end{array}$ & VAS & + & - \\
\hline Lee et al., 2014 & $\mathrm{RCT}$ & $\mathrm{SSNB}+\mathrm{ANB}$ vs. SSNB + placebo & VAS & - & - \\
\hline Lee et al., 2015 & $\mathrm{RCT}$ & Arthroscopy guided SSNB vs. placebo & VAS & + & - \\
\hline Lee et al., 2012 & Non-randomized & ISB vs. SSNB + ANB vs. none & VAS & + & - \\
\hline Lehmann et al., 2015 & $\mathrm{RCT}$ & GA vs. GA + ISB vs. ISB & NRS & + & + \\
\hline Merivirta et al., 2013 & $\mathrm{RCT}$ & $\begin{array}{l}\text { Subacromial bupivacaine infusion vs. } \\
\text { transdermal fentanyl patch }\end{array}$ & NRS & + & - \\
\hline Merolla et al., 2015 & $\mathrm{RCT}$ & Dietary supplement vs. placebo & VAS & + & - \\
\hline Park et al., 2016 & $\mathrm{RCT}$ & $\mathrm{SSNB}+\mathrm{ANB}$ vs. SSNB vs. none & VAS & - & - \\
\hline Perdreau et al., 2015 & $\mathrm{RCT}$ & Multimodal local injection vs. placebo & VAS & + & + \\
\hline Rubenis et al., 2015 & Non-randomized & $\begin{array}{l}\text { Undersurface rotator cuff repair vs. } \\
\text { bursal-side rotator cuff repair }\end{array}$ & Likert & - & - \\
\hline Ryu et al., 2015 & $\mathrm{RCT}$ & $\begin{array}{l}\text { Supraclavicular brachial plexus block } \\
\text { vs. ISB }\end{array}$ & NRS & + & + \\
\hline Salviz et al., 2013 & $\mathrm{RCT}$ & ISB vs. ISC vs. GA & NRS & + & + \\
\hline
\end{tabular}


Table 1. (continued).

\begin{tabular}{|c|c|c|c|c|c|}
\hline \multirow[b]{2}{*}{ Author, year } & \multirow[b]{2}{*}{ Study design } & \multirow[b]{2}{*}{ Intervention } & \multicolumn{3}{|c|}{ Pain-related outcomes } \\
\hline & & & Pain scale & $\begin{array}{l}\text { Analgesic } \\
\text { consumption }\end{array}$ & $\begin{array}{l}\text { Time to } \\
\text { first request }\end{array}$ \\
\hline Saritas et al., 2015 & $\mathrm{RCT}$ & $\begin{array}{l}\text { Intraarticular magnesium sulphate } \\
\text { vs. placebo }\end{array}$ & VAS & + & - \\
\hline Schwartzberg et al., 2013 & $\mathrm{RCT}$ & $\begin{array}{l}\text { Subacromial bupivacaine infusion vs. } \\
\text { placebo vs. none }\end{array}$ & VAS $^{* *}$ & + & - \\
\hline Shin et al., 2014 & $\mathrm{RCT}$ & $\begin{array}{l}\text { ISC infusion + PCA vs. ISC PCA vs. } \\
\text { IV PCA }\end{array}$ & NRS & + & - \\
\hline Shin et al., 2016 & $\mathrm{RCT}$ & C5-approach ISB vs. ISB vs. none & NRS & - & - \\
\hline Tham et al., 2013 & Cohort & $\begin{array}{l}\text { Correlation between tendon thickness } \\
\text { and pain }\end{array}$ & Likert & - & - \\
\hline Vorsanger et al., 2013 & $\mathrm{RCT}$ & Tapentadol vs. oxycodone & NRS & + & - \\
\hline Wei et al., 2014 & Up-and-down & Infusion rate of ropivacaine in ISC & NRS & + & - \\
\hline Wiegel et al., 2017 & $\mathrm{RCT}$ & SSNB vs. ISB & NRS & + & - \\
\hline Woo et al., 2014 & $\mathrm{RCT}$ & $\begin{array}{l}\text { Ketamine infusion during GA } \\
\text { combined with single-shot ISB }\end{array}$ & NRS & + & + \\
\hline Woo et al., 2015 & $\mathrm{RCT}$ & $\begin{array}{l}\text { Dexamethasone on the duration of } \\
\text { single shot ISB }\end{array}$ & NRS & + & + \\
\hline Yeo et al., 2017 & Cohort & $\begin{array}{l}\text { Correlation of rotator cuff tear area } \\
\text { and postoperative pain }\end{array}$ & Likert & - & - \\
\hline Yun et al., 2012 & $\mathrm{RCT}$ & $\begin{array}{l}\text { Patient-controlled analgesia vs. } \\
\text { intravenous patient-controlled analgesia }\end{array}$ & VAS & + & - \\
\hline
\end{tabular}

ANB, axillary nerve block; GA, general anesthesia; ISB, interscalene brachial plexus block; ISC, interscalene catheter; MIB, middle interscalene block; NRS, numeric rating scale; PCA, patient controlled analgesia; RCT, randomized controlled trial; VAS, visual analogue scale; SSNB, suprascapular nerve block.

* Cho et al. report using VAS but report that participants had to "point to the position on the line between the faces".

** Schwartszberg et al. report using a VAS, but describe pain scores being obtained verbally.

The VAS, NRS, and Likert scale all used similar minimum anchors such as "no pain". To describe higher pain levels, the scales used 14 different anchors in total (Figure 2) with "worst pain imaginable" $[11,12,15,26,36$, $38,43,48,51,54]$ being the preferred anchor. The second most frequent anchor was "worst pain" $[10,28,33,34]$ and then successively "most severe pain imaginable" [19,20,37], "severe pain" $[18,32,46]$, and "worst possible pain" $[29,41,47]$. Twelve studies did not report the used anchors [13,16,17,27,31,35,40,49,50,55-57].

Pain ratings were specified to be at rest or during activity in 11 studies [10,13,16,23,26-28,32,44,55,58], of which 3 studies $[10,16,26]$ specified pain to be exclusively measured at rest. Eight out of the 11 studies described pain during activity and the definitions were: movement during cough and mobilization [27], motion attempts [28], shoulder movements [58] during activities such as dressing or during transfer from lying to sitting [23], passive motion [55], during overhead activities and sleep [44]; two studies did not specify the activity [13,32]. The remaining 36 studies did not specify whether pain was measured during activity or at rest.

The following 6 studies asked patients to rate pain as either present pain, least pain, worst pain, or average pain during a specified period of time, namely average pain score for the day [25], daily average pain [40], overnight pain [29], level of pain during sleep [45], overall pain [32], and average of least and worst pain [13]. The remaining studies did not report these details of the pain recordings.

\section{Number of measurements and time points}

The total number of pain recordings within the first seven PODs was extracted from 45 studies (Figure 3); two studies did not report the total number of recordings $[15,54]$. The mean number of recordings within the first postoperative week was $6.3(0-21)$.

The distribution of recordings during the first seven PODs could be extracted from 45 studies (Figure 4). The majority of pain recordings were made on POD1, totaling $60 \%$ of cumulated number of recordings within the first postoperative week, equivalent to a mean of $5.1(0-11)$ recordings. The number of pain recordings decreased from POD2 to POD6 with a small increase in POD7. The time points in POD1 were specified in 32 of the included studies and represented 28 different time points (Figure 5) [10-12,14,19-21,23,26-28,30,31,33-38,43,46-52,54-57,59]. The most frequently used time points were: postanaesthesia care unit, 1, 2, 4, 6, 8, 12, and 24 hours postoperatively.

\section{Analgesic consumption}

In the 34 studies reporting analgesic consumption, different methods were used. Some studies used more than one method due to variations in analgesic protocols, such as different scheduled analgesics and rescue analgesics. In most cases, only the rescue analgesics were recorded (in mg 


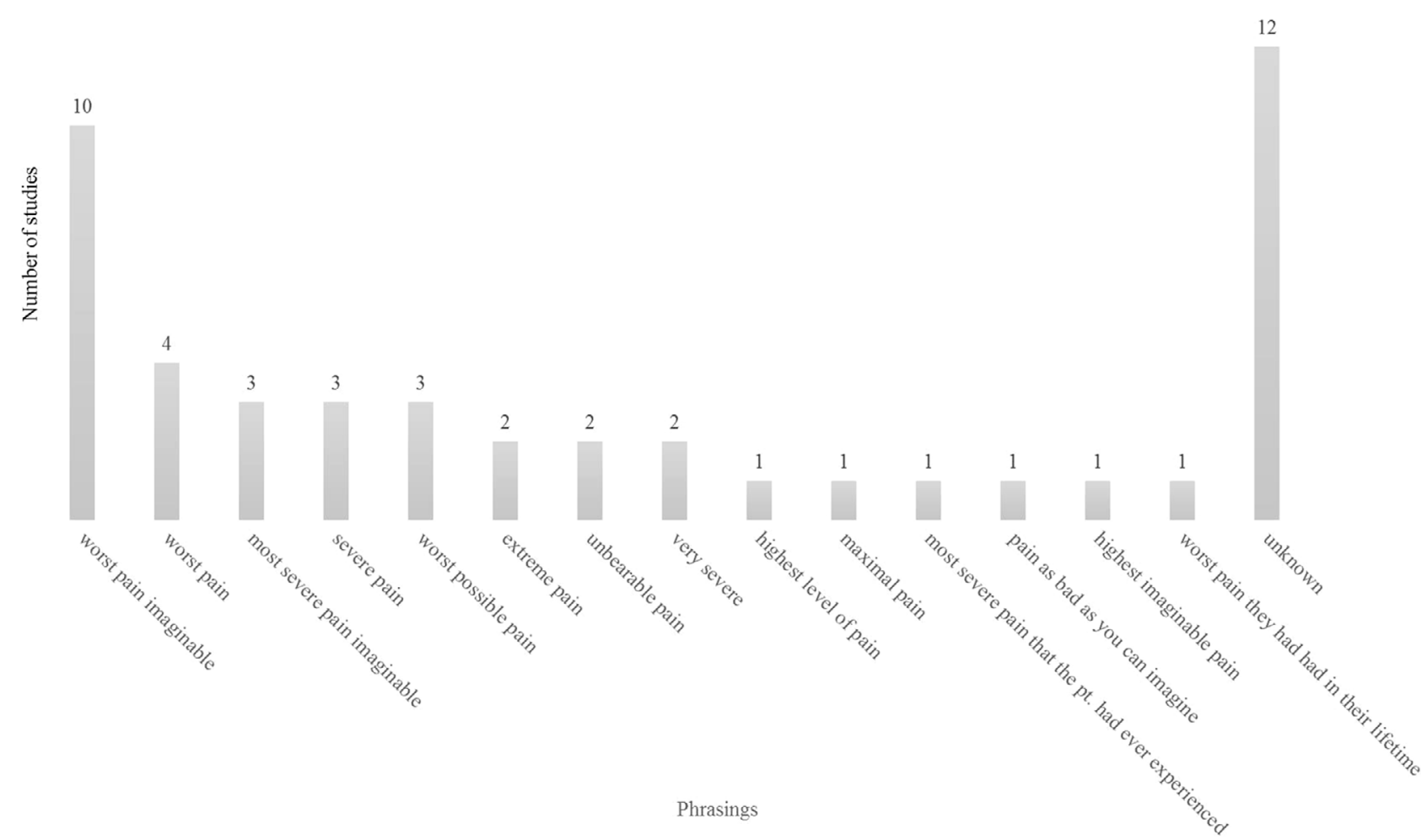

Figure 2. Maximum pain level anchors used $n=47$ studies. "Worst imaginable pain" was grouped with "worst pain imaginable".

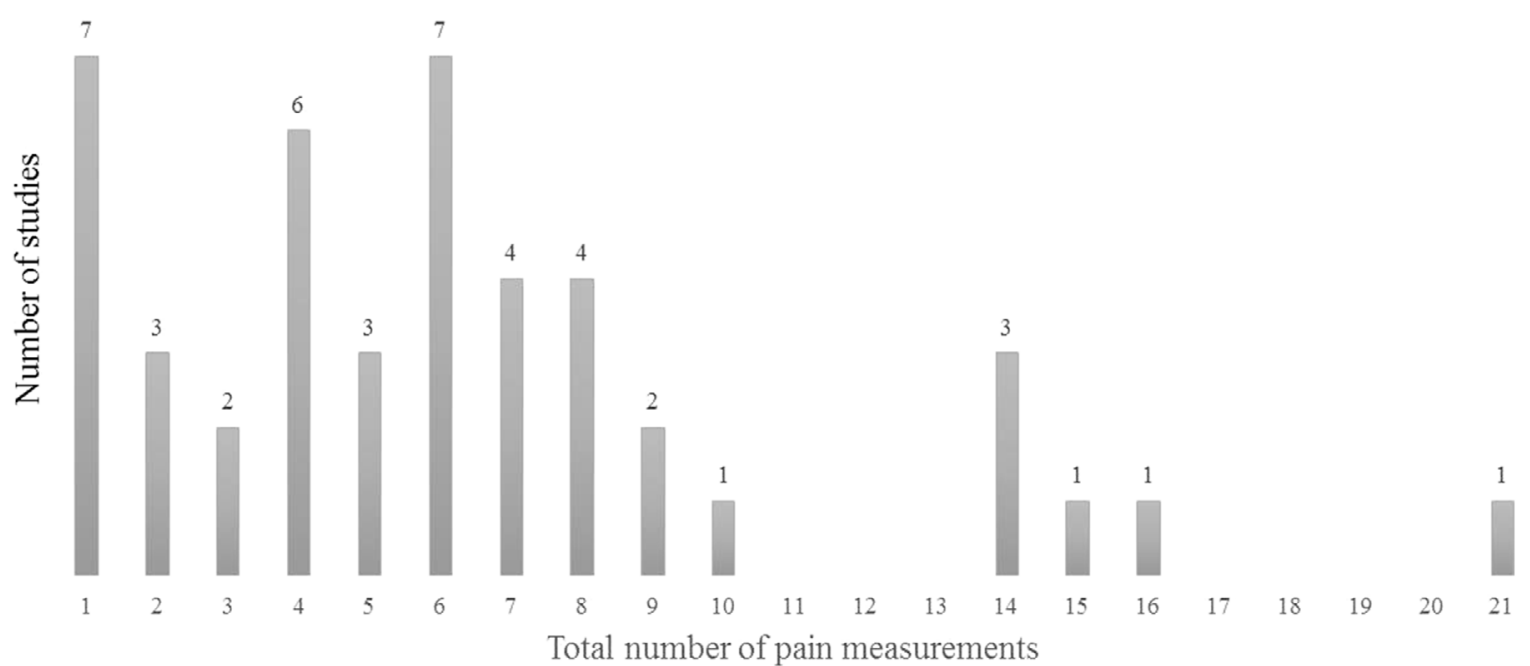

Figure 3. Total number of pain recordings during the first postoperative week $n=45$ studies.

or number of doses) $[14,21,24,47,49]$, or the number of patients requesting $[34,54,56]$ (or not requesting) analgesics [19]. For patients using patient-controlled analgesia (PCA, usually with intravenous opioid), the number of attempts, number of doses, and/or cumulated dose (for example during 24 hours) were reported $[31,37,41,48,50,52,57]$. Some studies reported total consumption of morphine/opioids [12,13,47] or cumulated amount of all analgesics $[10,15]$ during a specific period of time.

\section{Time to first analgesic request or first pain}

This was the least used primary pain outcome. Studies reporting this outcome were mainly, but not limited to, trials of peripheral nerve blocks. Four studies used time to first pain $[10,13,15,34]$, and seven studies used time to first request of analgesics [12,16-19,37,52]. Different starting points were used when reporting time to first analgesic request or first pain: time from injection of the local anesthetic solution [16,18], time from extubation [52], time 


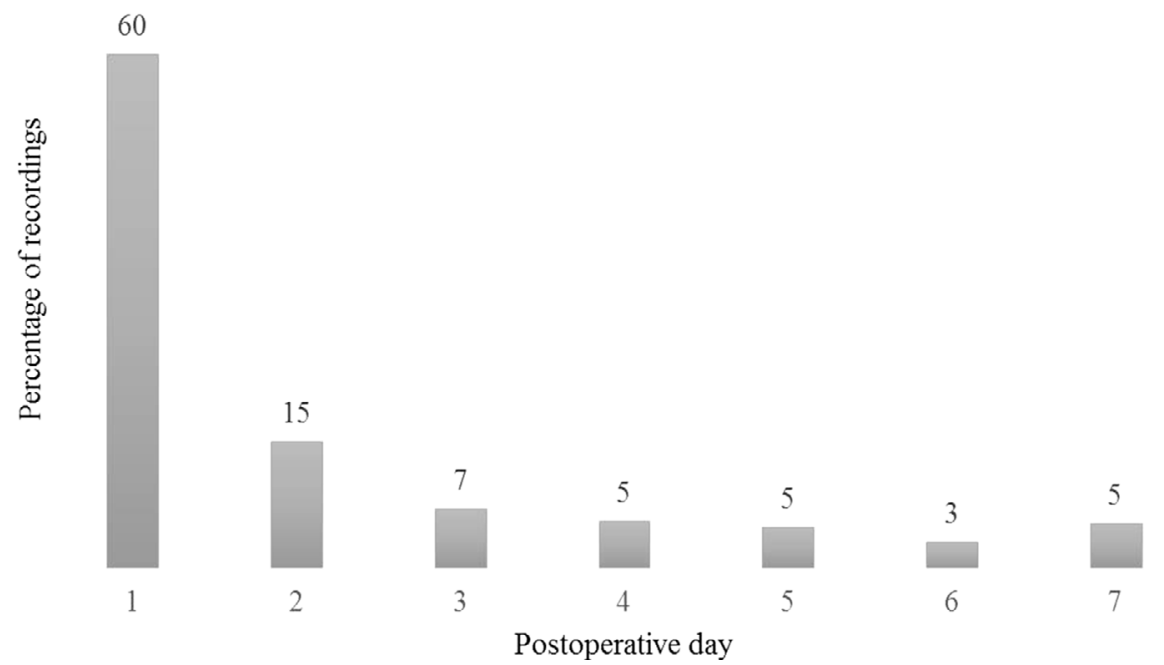

Figure 4. Percentage of pain recordings within the first seven postoperative days. The data represent 268 recordings, by any applied pain rating scale, extracted from 45 studies.

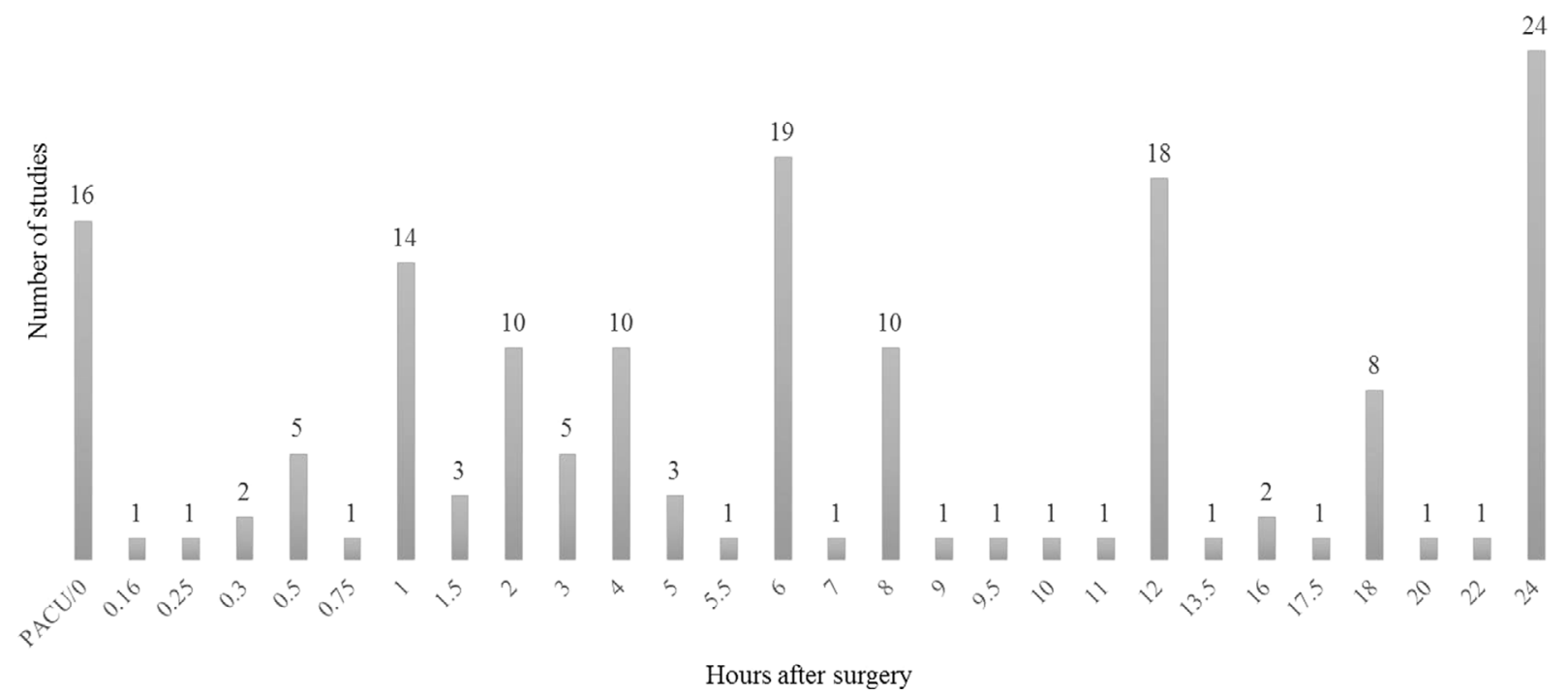

Figure 5. Number of studies using a specific time point, first 24-hour postoperative pain recordings. Data from 32 studies are included Q2 2 with a total of 162 measurements, equaling a mean of 5.1 pain recordings per study on POD1. *Erden et al. measured pain within time intervals $0-3,4-7,8-11,12-15,16-19,20-24$ hours. To include the data, the middle range was chosen as time of measurement. ** Jo et al. recorded pain 5 hours postoperatively and then three times daily (at 0:00 am, 9:00 am, and 5:00 pm). Since we do not know what time of day the patients were operated, only the first recording after 5 hours is included in the graph. ***Ryu et al. measured pain hourly until request of rescue analgesic which was requested at a mean of 11 hours and 45 minutes postoperatively on which basis we added these 11 pain recordings to the graph.

from closure [15], and time from arrival at the postanesthesia care unit [37].

\section{Discussion}

When screening the 711 abstracts for the current study, many of the first discarded 558 studies were found to use questionnaires with joint-specific or health-related quality of life composite scores (such as DASH score, American Shoulder and Elbow Surgeons (ASES) score, Oxford shoulder score, and Western Ontario Rotator Cuff Index).
Since data on pain assessment could not be extracted from these composite scores, these studies were unsuitable for further analysis of postoperative pain. Currently this issue is being investigated by others, for example Gagnier et al. [3] who has published a study protocol with the purpose of creating a core outcome set for clinical trials of people with shoulder pain as the lack of uniformity in outcome measures across clinical trials limits interstudy comparison and the ability to pool data for meta-analyses.

The majority of included studies used the VAS to measure pain intensity. The literature generally indicates 
that the VAS and the NRS are interchangeable, and some studies report coherence between the VAS and NRS pain rating scales $[4,60,61]$; others found no coherence at specific pain levels [62]. On basis of international guidelines, literature recommendations $[1,62,63,65]$, and practical applications where the NRS is seemingly easier to administer than the VAS $[63,64]$ (since it requires no remedies), this study recommends using the NRS to measure pain intensity.

Though the majority of included studies reported the chosen pain rating scale, such as the VAS or NRS, a description of scale use is rare to find, thus obscuring whether the scales were used as originally intended. In at least two of the included studies, the actual use of the VAS was described as a Faces Pain Scale and the NRS, respectively $[24,35]$.

The pain rating scales used 14 different anchors when phrasing maximum pain levels. Although there was little variation in the phrasing, the lack of consistency is noticeable. Hawker et al.'s [63] review article from 2011 reported that the VAS was designed using the anchor "severe pain", whereas the NRS was designed with the anchors "pain as bad as you can imagine" and "worst pain imaginable." Being able to compare future studies, it is important they apply the same scale and anchors, i.e., "no pain" and "worst pain imaginable."

Few studies reported if pain was measured during activity or at rest. Recording pain during activity increases sensitivity due to higher pain scores, and reflects patients' functional levels. Based on international recommendations $[1,65]$, it is recommended to report both pain at rest and during activity. Focus is thus drawn on pain during deep breathing and coughing to reduce risks of cardiopulmonary and thromboembolic complications after surgery [4].

The majority of pain recordings within the first postoperative week were recorded on POD1. When looking at the time points on POD1, 28 different time points were used in 32 studies. Naturally, some of the variation is a result of peaks of interest, reflecting different interventions. However, some standardization seems possible. It is thus recommended not to report "postanesthesia care unit" as a time point but rather to use the most common time points of $1,2,4,6,8,12$, and 24 hours, preferably starting from injection of local anesthetic or from extubation, dependent on the intervention investigated. Too many measurements will cause problems if not using the proper statistical methods, but this is beyond the scope of this review.

It is important to include the outcome of analgesic consumption, as the pain intensity ratings do not necessarily reflect the patients' wishes for additional opioids $[66,67]$. Some studies showed discrepancies between pain levels and analgesic consumption, with one outcome being statistically significant and the other not $[10,11,13,18,21$, $41,48,50,52]$. It is therefore advisable to report both analgesic intake as well as pain intensity ratings [68].
Reporting morphine equivalent doses, as was done in some studies, bears the risk of error in the conversion factors, but may be necessary if the protocol involves more than one opioid and facilitates interstudy comparison.

Time to first analgesic request or first pain is related to the "time to remedication," often used in studies with nerve blocks, illustrating when patients will need further attention [5]. Time to first pain would be more sensitive, as onset of pain may precede the first analgesic request, especially in patients reluctant to consume medication. It is important to use the recommended starting points here as well, such as time from injection or extubation.

\section{Limitations}

Despite a wide-ranging search strategy, the risk of missing relevant studies cannot be excluded. However, the considerable variation regarding outcomes and reported methods indicates that a comprehensive search was conducted. Also, two databases were searched for relevant literature, thus strengthening the study. It is assumed that the findings from the current study can be generalized to other populations with acute postoperative pain as there is no apparent reason to assume that postoperative pain following rotator cuff surgery is measured differently from other types of surgery, but this may be a subject for further investigations.

\section{Conclusion}

A large variety in the reporting of postoperative pain outcomes in the 47 included studies was shown. Based on this study and international guidelines, future postoperative pain trials are recommended to include the following outcomes: pain intensity using the NRS with the anchors "no pain" and "worst pain imaginable," recorded during activity and at rest at 1, 2, 4, 6, 8, 12, and 24 hours postoperatively. Analgesic consumption should also be included and reported as cumulated rescue analgesic medication in $\mathrm{mg}$, if possible converted to morphine equivalents.

Assuming that postoperative pain following rotator cuff surgery is not measured differently from other types of surgery, the applications of this study may be expanded to clinical trials involving patients undergoing other surgical procedures.

\section{Conflict of interest}

The authors declare that they have no conflicts of interest in relation to this article.

Acknowledgements. The authors thank Vicki Elisabeth Vallund (Department of Anaesthesiology and Intensive Care, Aarhus, Denmark) for English language revision. 


\section{References}

1. Rothaug J, Zaslansky R, Schwenkglenks M, Komann M, Allvin R, Backstrom R, et al. (2013) Patients' perception of postoperative pain management: validation of the International Pain Outcomes (IPO) questionnaire. J Pain 14, 13611370

2. Page MJ, McKenzie JE, Green SE, Beaton DE, Jain NB, Lenza M, et al. (2015) Core domain and outcome measurement sets for shoulder pain trials are needed: systematic review of physical therapy trials. J Clin Epidemiol 68, 1270-1281.

3. Gagnier JJ, Page MJ, Huang H, Verhagen AP, Buchbinder R (2017) Creation of a core outcome set for clinical trials of people with shoulder pain: a study protocol. Trials 18, 336 .

4. Breivik H, Borchgrevink P, Allen S, Rosseland L, Romundstad L, Hals E, et al. (2008) Assessment of pain. Br J Anaesth 101, 17-24.

5. Barden J, Edwards J, Mason L, McQuay H, Moore R (2004) Outcomes in acute pain trials: systematic review of what was reported? Pain 109, 351.

6. Williamson PR, Altman DG, Bagley H, Barnes KL, Blazeby JM, Brookes ST, et al. (2017) The COMET Handbook: Version 1.0. Trials 20; 18(Suppl 3), 280.

7. Bjørnholdt KT, Jensen JM, Bendtsen TF, Søballe K, Nikolajsen L (2014) Local infiltration analgesia versus continuous interscalene brachial plexus block for shoulder replacement pain: a randomized clinical trial. Eur J Orthop Surg Traumatol 25, 1245-1252.

8. Bjørnholdt KT, Mønsted PN, Søballe K, Nikolajsen L (2014) Dexamethasone for pain after outpatient shoulder surgery: a randomised, double-blind, placebo-controlled trial. Acta Anaesthesiol Scand 58, 751-758.

9. Covidence systematic review software, Veritas Health Innovation, Melbourne, Australia. Available at http:// www.covidence.org/.

10. Abdallah FW, Dwyer T, Chan VW, Niazi AU, OgilvieHarris DJ, Oldfield S, et al. (2016) IV and Perineural dexmedetomidine similarly prolong the duration of analgesia after interscalene brachial plexus block: a randomized, three-arm, triple-masked, placebo-controlled trial. Anesthesiology 124, 683-695.

11. Ahn S, Byun SH, Park K, Ha JL, Kwon B, Kim JC (2016) Analgesic efficacy of preemptive pregabalin administration in arthroscopic shoulder surgery: a randomized controlled trial. Can J Anaesth 63, 283-189.

12. Aksu R, Biçer C, Ülgey A, Bayram A, Günes I, Güney A, et al. (2015) Comparison of interscalene brachial plexus block and intra-articular local anesthetic administration on postoperative pain management in arthroscopic shoulder surgery. Braz J Anesthesiol 65, 222-229.

13. Cheng J, Kahn RL, YaDeau JT, Tsodikov A, Goytizolo EA, Guheen CR, et al. (2016) The fibromyalgia survey score correlates with preoperative pain phenotypes but does not predict pain outcomes after shoulder arthroscopy. Clin J Pain 32, 689-694.

14. Cho CH, Lee SW, Lee YK, Shin HK, Hwang I (2015) Effect of a sleep aid in analgesia after arthroscopic rotator cuff repair. Yonsei Med J 56, 772-777.

15. Lehmann LJ, Loosen G, Weiss C, Schmittner MD (2015) Interscalene plexus block versus general anaesthesia for shoulder surgery: a randomized controlled study. Eur J Orthop Surg Traumatol 25, 255-261.

16. Alemanno F, Ghisi D, Westermann B, Bettoni A, Fanelli A, La Colla L, et al. (2016) The use of vitamin B1 as a perineural adjuvant to middle interscalene block for postoperative analgesia after shoulder surgery. Acta Biomed 87, 22-27.

17. Alemanno F, Westermann B, Bettoni A, Candiani A, Cesana BM (2014) Buprenorphine versus tramadol as perineural adjuvants for postoperative analgesia in patients undergoing arthroscopic rotator cuff repair under middle interscalene block: a retrospective study. Minerva Anestesiol 80, 1198-1204.

18. Desmet M, Vanneste B, Reynvoet M, Van Cauwelaert J, Verhelst L, Pottel H, et al. (2015) A randomised controlled trial of intravenous dexamethasone combined with interscalene brachial plexus blockade for shoulder surgery. Anaesthesia 70, 1180-1185.

19. Woo JH, Kim YJ, Kim DY, Cho S (2015) Dose-dependency of dexamethasone on the analgesic effect of interscalene block for arthroscopic shoulder surgery using ropivacaine 0.5\%: a randomised controlled trial. Eur J Anaesthesiol 32, 650-655.

20. Choi EM, Kim EM, Chung MH, Park JH, Lee HK, Choi YR, et al. (2015) Effects of ultrasound-guided stellate ganglion block on acute pain after arthroscopic shoulder surgery. Pain Physician 18, E379- E388.

21. Han SS, Lee YH, Oh JH, Aminzai S, Kim SH (2013) Randomized, controlled trial of multimodal shoulder injection or intravenous patient-controlled analgesia after arthroscopic rotator cuff repair. Knee Surg Sports Traumatol Arthrosc 21, 2877-2883.

22. Zhai W, Wang X, Rong Y, Li M, Wang H (2016) Effects of a fixed low-dose ropivacaine with different volume and concentrations on interscalene brachial plexus block: a randomized controlled trial. BMC Anesthesiol 16, 80.

23. Alfuth M, Strietzel M, Vogler T, Rosenbaum D, Liem D (2016) Cold versus cold compression therapy after shoulder arthroscopy: a prospective randomized clinical trial. Knee Surg Sports Traumatol Arthrosc 24, 2209-2215.

24. Cho CH, Song KS, Min BW, Jung GH, Lee YK, Shin HK (2015) Efficacy of interscalene block combined with multimodal pain control for postoperative analgesia after rotator cuff repair. Knee Surg Sports Traumatol Arthrosc $23,542-547$.

25. Cuff DJ, O'Brien KC, Pupello DR, Santoni BG (2016) Evaluation of factors affecting acute postoperative pain levels after arthroscopic rotator cuff repair. Arthroscopy 32, 1231-1236.

26. Dhir S, Sondekoppam RV, Sharma R, Ganapathy S, Athwal GS (2016) A comparison of combined suprascapular and axillary nerve blocks to interscalene nerve block for analgesia in arthroscopic shoulder surgery: an equivalence study. Reg Anesth Pain Med 41, 564-571.

27. Erden S, Demir SG, Kanatlı U, Danacı F, Carboga B (2017) The effect of standard pain assessment on pain and analgesic consumption amount in patients undergoing arthroscopic shoulder surgery. Appl Nurs Res 33, 121-126.

28. Kim JY, Song KS, Kim WJ, Park YH, Kang H, Woo YC, et al. (2016) Analgesic efficacy of two interscalene blocks and one cervical epidural block in arthroscopic rotator cuff repair. Knee Surg Sports Traumatol Arthrosc 24, 931-939.

29. Lane S, Blundell C, Mills S, Charalambous CP (2014) Same day discharge following inter-scalene block administration for arthroscopic shoulder surgery: implementing a change in practice. J Perioper Pract 24, 232-234.

30. Lee JJ, Kim DY, Hwang JT, Lee SS, Hwang SM, Kim GH, et al. (2014) Effect of ultrasonographically guided axillary nerve block combined with suprascapular nerve block in arthroscopic rotator cuff repair: a randomized controlled trial. Arthroscopy 30, 906-914. 
31. Lee SM, Park SE, Nam YS, Han SH, Lee KJ, Kwon MJ, et al. (2012) Analgesic effectiveness of nerve block in shoulder arthroscopy: comparison between interscalene, suprascapular and axillary nerve blocks. Knee Surg Sports Traumatol Arthrosc 20, 2573-2578.

32. Merolla G, Dellabiancia F, Ingardia A, Paladini P, Porcellini G (2015) Co-analgesic therapy for arthroscopic supraspinatus tendon repair pain using a dietary supplement containing Boswellia serrata and Curcuma longa: a prospective randomized placebo-controlled study. Musculoskelet Surg 99, 43-52.

33. Park JY, Bang JY, Oh KS (2016) Blind suprascapular and axillary nerve block for post-operative pain in arthroscopic rotator cuff surgery. Knee Surg Sports Traumatol Arthrosc $24,3877-3883$.

34. Salviz EA, Xu D, Frulla A, Kwofie K, Shastri U, Chen J, et al. (2013) Continuous interscalene block in patients having outpatient rotator cuff repair surgery: a prospective randomized trial. Anesth Analg 117, 1485-1492.

35. Schwartzberg RS, Reuss BL, Rust R (2013) Efficacy of continuous subacromial bupivacaine infusion for pain control after arthroscopic rotator cuff repair. J Shoulder Elbow Surg 22, 1320-1324.

36. Wiegel M, Moriggl B, Schwarzkopf P, Petroff D, Reske AW (2017) Anterior suprascapular nerve block versus interscalene brachial plexus block for shoulder surgery in the outpatient setting: a randomized controlled patientand assessor-blinded trial. Reg Anesth Pain Med 42, $310-318$.

37. Woo JH, Kim YJ, Baik HJ, Han JI, Chung RK (2014) Does intravenous ketamine enhance analgesia after arthroscopic shoulder surgery with ultrasound guided single-injection interscalene block?: a randomized, prospective, doubleblind trial. J Korean Med Sci 29, 1001-1006.

38. Wei Y, Li M, Rong Y, Guo X (2014) Effective background infusion rate of ropivacaine $0.2 \%$ for patient-controlled interscalene brachial plexus analgesia after rotator cuff repair surgery. Chin Med J 127, 4119-4123.

39. Vorsanger GJ, Klopfer AM, Xiang J, Benson CJ, Moskovitz BL, Rosenthal NR (2013) Immediate-release tapentadol or oxycodone for treatment of acute postoperative pain after elective arthroscopic shoulder surgery: a randomized, phase IIIb study. J Opioid Manag 9, 281-290.

40. D'Ambrosi R, Palumbo F, Paronzini A, Ragone V, Facchini RM (2016) Platelet-rich plasma supplementation in arthroscopic repair of full-thickness rotator cuff tears: a randomized clinical trial. Musculoskeletal Surg 100, 25-32.

41. Khashan M, Dolkart O, Amar E, Chechik O, Sharfman Z, Mozes G, et al. (2016) Effect of preemptive intra-articular morphine and ketamine on pain after arthroscopic rotator cuff repair: a prospective, double-blind, randomized controlled study. Arch Orthop Trauma Surg 136, 233239.

42. Kraeutler MJ, Reynolds KA, Long C, McCarty EC (2015) Compressive cryotherapy versus ice-a prospective, randomized study on postoperative pain in patients undergoing arthroscopic rotator cuff repair or subacromial decompression. J Shoulder Elbow Surg 24, 854-859.

43. Shin HJ, Na HS, Oh AY, Hwang JW, Kim BG, Park HP, et al. (2016) A prospective, randomized and controlled study of interscalene brachial plexus block for arthroscopic shoulder surgery: a comparison of C5 and conventional approach, a CONSORT-compliant article. Medicine 95, e4921.

44. Tham ER, Briggs L, Murrell GA (2013) Ultrasound changes after rotator cuff repair: is supraspinatus tendon thickness related to pain? J Shoulder Elbow Surg 22, e8-e15.
45. Yeo DYT, Walton JR, Lam P, Murrell GAC (2017) The relationship between intraoperative tear dimensions and postoperative pain in 1624 consecutive arthroscopic rotator cuff repairs. Am J Sports Med 45, 788-793.

46. Basat H, Uçar DH, Armangil M, Güçlü B, Demirtas M (2016) Post operative pain management in shoulder surgery: Suprascapular and axillary nerve block by arthroscope assisted catheter placement. Indian J Orthop 50, 584589.

47. Faria-Silva R, de Rezende DC, Ribeiro JM, Gomes TH, Oliveira BA, Pereira FM, et al. (2016) Association of clonidine and ropivacaine in brachial plexus block for shoulder arthroscopy. Braz J Anesthesiol 66, 335-340.

48. Jo CH, Shin JS, Huh J (2014) Multimodal analgesia for arthroscopic rotator cuff repair: a randomized, placebocontrolled, double-blind trial. Eur J Orthop Surg Traumatol $24,315-322$.

49. Lee HJ, Kim YS, Park I, Ha DH, Lee JH (2015) Administration of analgesics after rotator cuff repair: a prospective clinical trial comparing glenohumeral, subacromial, and a combination of glenohumeral and subacromial injections. J Shoulder Elbow Surg 24, 663-668.

50. Lee JJ, Yoo YS, Hwang JT, Kim DY, Jeon SJ, Hwang SM, et al. (2015) Efficacy of direct arthroscopy-guided suprascapular nerve block after arthroscopic rotator cuff repair: a prospective randomized study. Knee Surg Sports Traumatol Arthrosc 23, 562-566.

51. Merivirta R, Äärimaa V, Aantaa R, Koivisto M, Leino K, Liukas A, et al. (2013) Postoperative fentanyl patch versus subacromial bupivacaine infusion in arthroscopic shoulder surgery. Arthroscopy 29, 1129-1134.

52. Perdreau A, Joudet T (2015) Efficacy of multimodal analgesia injection combined with corticosteroids after arthroscopic rotator cuff repair. Orthop Traumatol Surg Res 101, 337-345.

53. Rubenis I, Lam PH, Murrell GA (2015) Arthroscopic rotator cuff repair using the undersurface technique: a 2 -year comparative study in 257 patients. Orthop J Sports Med 3, 1-9.

54. Ryu T, Kil BT, Kim JH (2015) Comparison between ultrasound-guided supraclavicular and interscalene brachial plexus blocks in patients undergoing arthroscopic shoulder surgery: a prospective, randomized, parallel study. Medicine 94, e1726.

55. Saritas TB, Borazan H, Okesli S, Yel M, Otelcioglu S; (2015) Is intra-articular magnesium effective for postoperative analgesia in arthroscopic shoulder surgery? Pain Res Manag $20,35-38$.

56. Shin SW, Byeon GJ, Yoon JU, Ok YM, Baek SH, Kim KH, et al. (2014) Effective analgesia with ultrasound-guided interscalene brachial plexus block for postoperative pain control after arthroscopic rotator cuff repair. J Anesth 28, 64-69.

57. Yun MJ, Oh JH, Yoon JP, Park SH, Hwang JW, Kil HY (2012) Subacromial patient-controlled analgesia with ropivacaine provides effective pain control after arthroscopic rotator cuff repair. Knee Surg Sports Traumatol Arthrosc 20, 1971-1977.

58. Wiesmann T, Feldmann C, Müller HH, Nentwig L, Beermann A, El-Zayat BF, et al. (2016) Phrenic palsy and analgesic quality of continuous supraclavicular vs. interscalene plexus blocks after shoulder surgery. Acta Anaesthesiol Scand 60, 1142-1151.

59. Kim BG, Han JU, Song JH, Yang C, Lee BW, Baek JS (2017) A comparison of ultrasound-guided interscalene and supraclavicular blocks for post-operative analgesia after shoulder surgery. Acta Anaesthesiol Scand 61, 427-435. 
60. Ahlers SJ, Van Gulik L, Van Der Veen AM, Van Dongen H, Bruins P, Belitser S, et al. (2008) Comparison of different pain scoring systems in critically ill patients in a general ICU. Crit Care 12, 15.

61. Bahreini M, Jalili M, Moradi-Lakeh M (2015) A comparison of three self-report pain scales in adults with acute pain. J Emerg Med 48, 10-18.

62. Hartrick CT, Kovan J, Shapiro S (2003) The numeric rating scale for clinical pain measurement: a ratio measure? Pain Pract 3, 310-316.

63. Hawker GA, Mian S, Kendzerska T, French M (2011) Measures of adult pain: Visual Analog Scale for Pain (VAS Pain), Numeric Rating Scale for Pain (NRS Pain), McGill Pain Questionnaire (MPQ), Short-Form McGill Pain Questionnaire (SF-MPQ), Chronic Pain Grade Scale (CPGS), Short Form-36 Bodily Pain Scale (SF-36 BPS), and Measure of Intermittent and Constant Osteoarthritis Pain (ICOAP). Arthritis Care Res 63, 240-252.

64. Goransson KE, Heilborn U, Selberg J, von Scheele S, Djarv T (2015) Pain rating in the ED-a comparison between 2 scales in a Swedish hospital. Am J Emerg Med 33, 419-422.

65. Gordon DB, Polomano R, Pellino T, Turk D, McCracken L, Sherwood G, et al. (2010) Revised American Pain Society Patient Outcome Questionnaire (APS-POQ-R) for quality improvement of pain management in hospitalized adults: preliminary psychometric evaluation. J Pain 11, 1172-1186.

66. van Boekel RLM, Vissers KCP, van der Sande R, Bronkhorst E, Lerou JGC, Steegers MAH (2017) Moving beyond pain scores: Multidimensional pain assessment is essential for adequate pain management after surgery. PLoS One 12, e0177345.

67. van Dijk JF, Kappen TH, Schuurmans MJ, van Wijck AJ (2015) The relation between patients' NRS pain scores and their desire for additional opioids after surgery. Pain Pract 15, 604-609.

68. Juul RV, Knosgaard KR, Olesen AE, Pedersen KV, Kreilgaard M, Christrup LL, et al. (2016) A model-based approach for joint analysis of pain intensity and opioid consumption in postoperative pain. AAPS J 18, 1013-1022.

Cite this article as: Rasmussen JK, Nikolajsen L, Bjørnholdt KT (2018) Acute postoperative pain after arthroscopic rotator cuff surgery: A review of methods of pain assessment. SICOT-J, 4, 49. 